\title{
Wireless Sensing with Smart Skins
}

\author{
Vasileios Lakafosis, Xiaohua Yi, Taoran Le, Edward Gebara, Yang Wang, and Manos M. Tentzeris \\ Georgia Institute of Technology, Atlanta, GA, USA \\ vasileios@gatech.edu
}

\begin{abstract}
The ever-increasing need for perpetual ubiquitous cognition of our environment prompts the integration of numerous unobtrusive, extremely low-cost and passively powered wireless sensors into our surroundings. Smart skins, i.e. thin layers of modified materials on top of surfaces that surround our every-day lives, constitute ideal such sensor candidates for the ubiquitous awareness vision. In this paper we are presenting three different types of highly sensitive smart skin sensors for identity and genuineness authentication and seal proof, large metallic structural strain and crack detection and chemical gas sensing of ammonia and nitrogen dioxide. These low-profile smart skin prototypes share not only all the aforementioned desired characteristics but also exhibit high levels of accuracy and reliability in a flexible and rugged design.
\end{abstract}

\section{INTRODUCTION}

The need to perpetually sense different elements of our surrounding environment makes its presence in an everincreasing number of diverse applications. Moreover, this ubiquitous cognition has to be achieved not only with the least obtrusive techniques but also in the most effective way, both accuracy- and power-wise. Toward that goal we are investigating novel sensors that are battery-less, wireless, thin enough to be part of product or infrastructure surfaces, and at the same time, very low-cost. These so-called smart skins can be thought of as a new generation of novel sensors that are realized as thin layers of modified materials that differ based on the intended application and that are massively deployed all around the body of very large structures, such as aircrafts, vehicles and bridges.

In this paper, we are presenting such highly sensitive smart skin sensors, which are targeted for three different essential applications:

- Identity authentication and seal proof: smart skins of this kind have the ability to authenticate an identity, serve as certificate of authenticity ( $\mathrm{CoA})$ and/or proof that a bottle or envelope is totally sealed

- Metallic structural strain detection: fatigue-induced fractures and cracks are the most important problems that metallic bridges and other large structures can experience over their lifetime and smart skins of this category are designed to accurately detect them

- Chemical gas sensing: dangerous industrial-grade gases, such as ammonia and nitrogen dioxide, must be detected early enough and at very low quantities to ensure timely response and avoid human loss

\section{IDENTITY AUTHENTICATION AND SEAL PROOF}

An arbitrary constellation of small, thin pieces of metallic conductors and/or one or more discrete dielectric materials and meta-materials (materials that exhibit a negative index of refraction) that are randomly dispersed and spatially fixed into an electromagnetic (EM) wave permeable dielectric fixative can behave as certificates of authenticity and seal proof. The idea is that these modified smart skins yield a hard-to-predict and unique signature of scattering parameters when brought within the near field, both reactive and radiating, of a reader's antenna array [1].

A very simple example of an authentication and seal proof smart skin certificate is shown in Fig. 1a. The fabrication process of this particular certificate involves the encapsulation of copper wire of variable gauge into a $2 \mathrm{~cm} \times 2 \mathrm{~cm} \times 1 \mathrm{~mm}$ plastic mold. Albeit its very simple and cheap structure, the yielded signature, depicted in Fig. 1b, exhibits distinct and strong variance of the transmission parameters when placed in front of 72 different transmitter and receiver antennae couplings that are extracted by our custom fabricated reader in the 5 to $6 \mathrm{GHz}$ frequency range.

Our reader's board circuit design is shown in Fig. 2, the major analog and digital components of which are annotated. The main objectives of this design have been, first, to maximize the entropy, i.e. randomness, of the EM fingerprint,

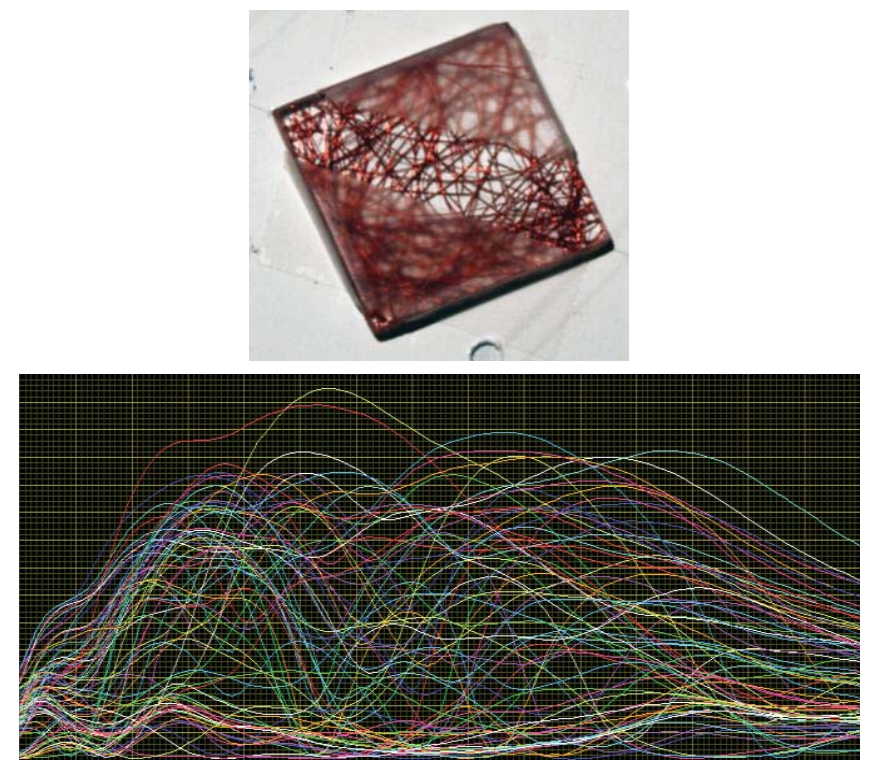

Fig. 1. (a) Copper-based smart skin certificate, (b) Graphical representation of a smart skin certificate signature as extracted by our fabricated reader for all its 72 antenna element couplings. 
given the accuracy of the analog and digital circuitry used, as well as the noise introduced by external factors and, second, to exhibit fingerprint consistency across multiple readings of the same smart skin, as well as across readings of the same smart skin by different readers. The board occupies a 3.85 in $x 7.8$ in area and consists of four metallic and three substrate layers, the total thickness of which does not exceed $1.6 \mathrm{~mm}$. The substrate of the board is FR-408. The antenna array consists of 25 folded microstrip patch antenna elements with a resonant frequency of $5.15 \mathrm{GHz}$.

The extraction of the unique EM fingerprint of a certificate is performed as follows. EM signal power is radiated from a particular element of the antenna array, then it is scattered and reflected by the conductive material of the instance placed a few $\mathrm{mm}$ away from the array and, finally, it is received by another antenna element. Each one of the curves of the EM fingerprint corresponds to a single coupling of a pair of antenna elements that is selected by digitally controlling RF switches with the micro-controller unit (MCU). The latter's $\mathrm{ADC}$ is also used for the readout, during which it is ensured that the placement of the CoA instance is fixed and geometrically unique, using short plastic poles, the relative position of which is non-symmetrical on the array's plane.

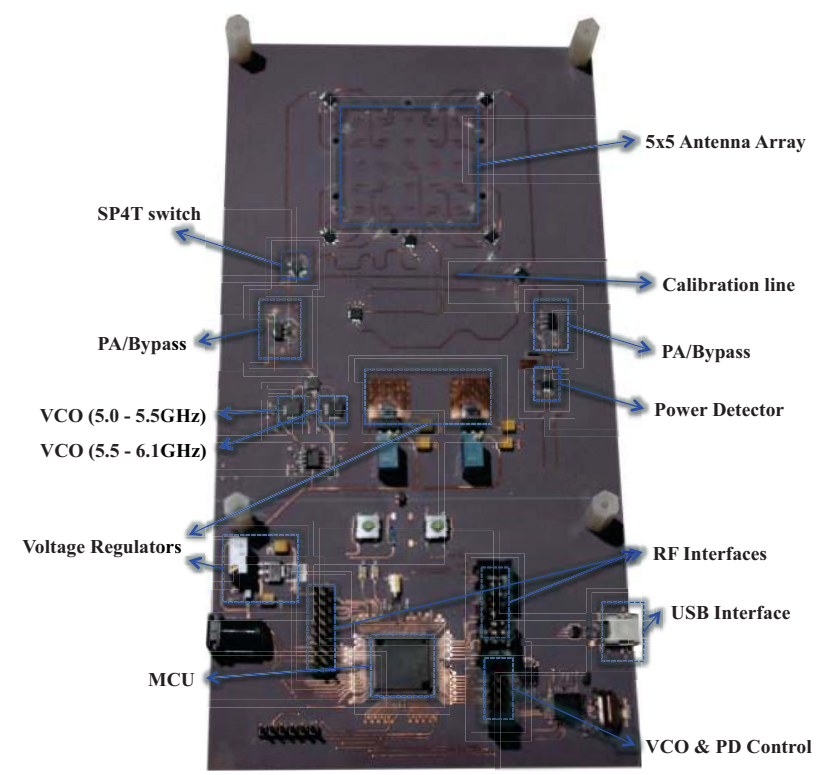

Fig. 2. The standalone, micro-controller-enabled, fabricated smart skin certificate reader.

The uniqueness, or entropy, among different fingerprints extracted from different $\mathrm{CoA}$ designs by our prototype reader is represented by the near-field frequency response for 10 different CoA objects across 4 different $T_{x}-R_{x}$ couplings, shown in Fig. 3. In this graph, the $y$-axis corresponds to the standard deviation of the received signal strength of the nearfield response captured by the reader, and the $\mathrm{x}$-axis corresponds to the different frequency points sampled by the MCU's ADC. The fact that the dynamic range of standard deviation, i.e. the square root of the estimator of the variance of the signal strength received by the $\mathrm{R}_{\mathrm{x}}$ element for different CoAs at the same frequency points and antenna couplings, is $45 \mathrm{dBm}$ shows our reader's superior acumen and our system's potential.

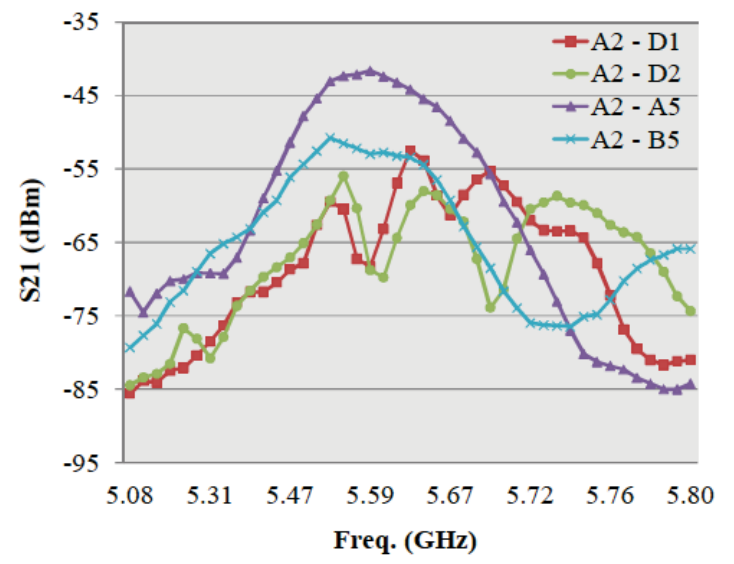

Fig. 3. Standard deviation of 10 different $S_{21}$ curves (of 10 different CoAs) for 4 antenna couplings.

These very thin physical certificates can be integrated onto the surfaces or openings of various objects. From the authenticity point of view, each certificate instance can be associated with an object, the authenticity of which the issuer wants to vouch, by storing its unique signature into the chip of any typical, passive or not, RFID tag [2]. They can also serve as seal proofs since these smart skins can be part of the tape of various object openings, such as bottles, envelopes, etc. When these protective tapes are even slightly torn the near-field EM signature extracted is not any more the same. As for the related cost, not only is the production cost of these random unique physical structures comparable to the price of typical passive RFID tags (few cents of dollars) but also their uniqueness incurs prohibitively high expenses for an adversary to even nearly reproduce them.

\section{Metallic Structural Strain Detection}

Fatigue-induced fractures and cracks are among the most common concerns for inspectors of large metallic constructions, such as highway bridges. The low-profile design that serves here as smart skin strain sensor is a quarterwave rectangular folded patch antenna that adopts a specially chosen substrate material with low-dielectric attenuation, as well as an inexpensive off-the-shelf RFID chip for signal modulation. The basic concept is to exploit the straindependent behavior of the antenna radiation as the sensing mechanism [3]. In particular, when a small piece of 2D antenna is under strain/deformation, its resonance frequency may change accordingly, which can be wirelessly interrogated by an RFID reader.

A thicker substrate enables a longer interrogation range, but reduces the strain transferred through the substrate (i.e. from the underlying structure, where the back of the substrate is bonded on, to the front-side copper cladding). This balance has been considered during the tag design. The fabricated smart skin strain sensor is shown in Fig. 4 . A 61 by 69 by 0.79 $\mathrm{mm}$ glass microfiber reinforced poly-tetra-fluoro-ethylene composite material is chosen as the antenna substrate, on the top and bottom of which are located the $0.0178 \mathrm{~mm}$-thick copper antenna and ground layers. Vias through the substrate are used for connecting the top copper cladding with the ground plane on the back of the substrate, as well as connecting one pin of the IC chip with the ground plane. 

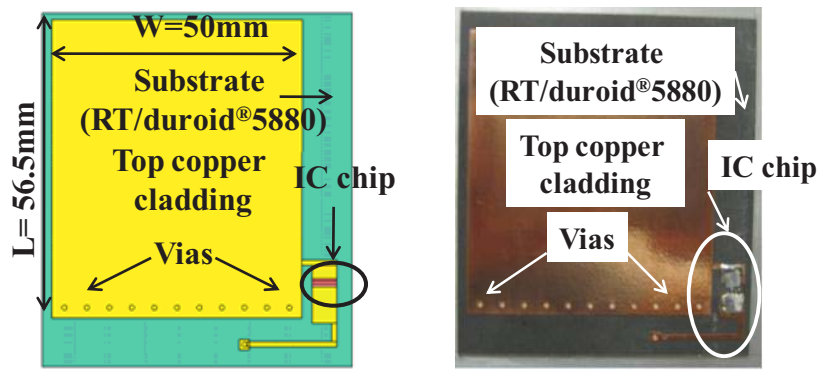

Fig. 4. The (a) designed and (b) fabricated RFID-enabled prototype smart skin strain sensors.

For the tensile testing experiments, the fabricated smart skin sensor is attached at the center of an aluminum specimen, which is shown in Fig. 5a. Fig. 5b shows the experimental setup for the tensile testing with a 22-kip SATEC machine. The reader antenna is mounted on a tripod facing the wireless strain sensor. The distance between the reader antenna and the wireless sensor is set as $12 \mathrm{in}$. Through a coaxial cable, the reader antenna is connected with a Tagformance reader. A strain gage module (NI 9235), in combination with a CompactDAQ Chassis (NI cDAQ-9172), is used for collecting data from conventional metal foil strain gages.

The specimen together with the smart skin strain sensor is stretched gradually up to $200 \mu \varepsilon$, at $\sim 20 \mu \varepsilon$ steps. The average transmitted power threshold levels for the sensor are shown in Fig. 6a. For clarity, only three example strain levels are shown in the figure. The mapping of the resonance frequency to the corresponding strain level is depicted in Fig. $6 \mathrm{~b}$. The ten data points correspond to ten strain levels. As a result of linear regression, the slope parameter is identified as $-726 \mathrm{~Hz} / \mu \varepsilon$ (i.e. $-0.000726 \mathrm{MHz} / \mu \varepsilon)$. This parameter corresponds to the strain sensitivity of the smart skin strain sensor, which means that 1 $\mu \varepsilon$ increment in the aluminum specimen causes $726 \mathrm{~Hz}$ decrease in the resonance frequency of the smart skin strain sensor. Fig. $6 \mathrm{~b}$ also shows the coefficient of determination, $\mathrm{R}^{2}$, from the linear regression. A value for $\mathrm{R}^{2}$ of 0.9915 indicates a good level of linearity. It is shown that the proposed smart skin strain sensor can detect strain changes lower than $20 \mu \varepsilon$.

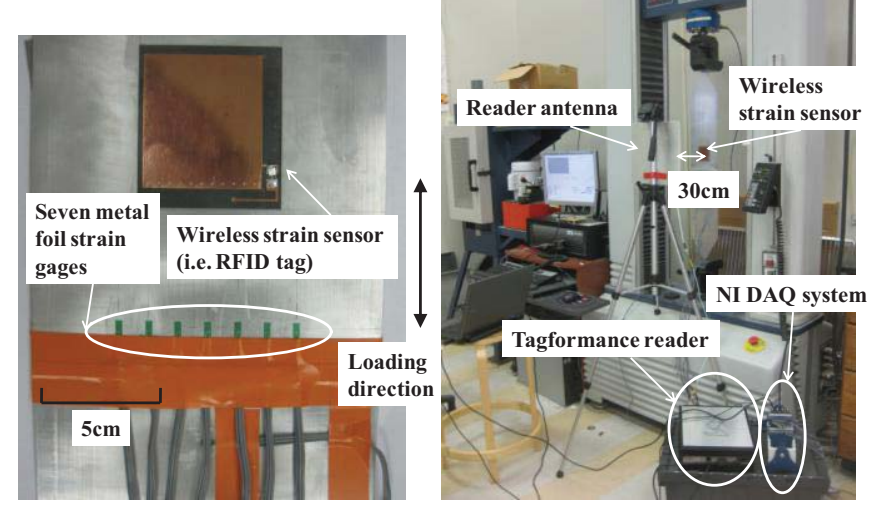

Fig. 5. (a) Sensor instrumentation, (b) Tensile tests setup

\section{Chemical Gas Sensing}

Carbon nanotubes (CNTs) alter their material properties, in particular the load permittivity, conductivity and impedance when in the presence of a given chemical substance, such as
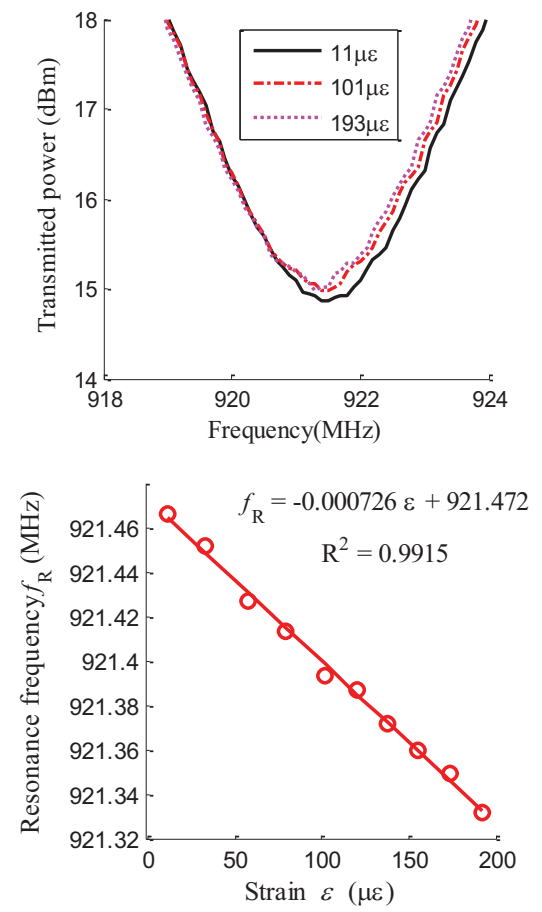

Fig. 6. Tensile testing results for the $\sim 20 \mu \varepsilon /$ step loading case at an interrogation distance of 12 in. (a) Average transmitted power threshold, (b) Resonance frequency $f_{\mathrm{R}}$ versus strain $\varepsilon$.

ammonia $\left(\mathrm{NH}_{3}\right)$ or nitrogen dioxide $\left(\mathrm{NO}_{2}\right)$. By integrating functionalized CNT materials onto antennas and other passive structures, we can translate the changes in material property into measurable quantities, such as shifts in resonant frequency, backscatter amplitude and voltage and/or current changes. In addition, the sensitivity of the material can be amplified by appropriate design choices. In the following, we present the use of multi-walled CNTs (MWCNT) for the purpose of gas sensing. In addition, instead of using the conventional highly toxic and cancerous organic compound Dimethylformamide (DMF) as a solvent for CNTs, we introduce the first ever water soluble MWCNT for inkjet printing applications. As a result, not only do we achieve excellent sensitivity results with water and sonication of MWCNTs, but also this latter solvent is much cheaper and easier to produce compared to single-walled CNTs.

The MWCNT material is produced via chemical vapor deposition (CVD), treated with acid to purify, and is functionalized to allow for better solubility in water. Glycol is added to the solution to increase the viscosity of the material and enhance flow through the printer. Immediately prior to starting the printing process, the mixture is sonicated for 1 hour and subsequently degassed for the same duration. The resulting mixture is deposited onto low-cost paper substrate via a Dimatix DMCLCP-11610 printer head using $10 \mathrm{pL}$ cartridges. To the best of our knowledge, this is the first time that MWCNT material is being delivered via inkjet printing and the first time water is used as the solvent.

To test the material, short microstrip MWCNT transmission lines (TL) of $2 \mathrm{~mm}$ by $3 \mathrm{~mm}$ deposited in between silver inkjet printed traces were used. Such structures with CNT depositions of 25, 50,75, and 100 layers are shown in Fig. 7. $\mathrm{NH}_{3}$ and $\mathrm{NO}_{2}$, diluted in nitrogen $\left(\mathrm{N}_{2}\right)$, were chosen as test gases due to their extensive industrial use, toxicity, and relative ease of obtainment. The effect of these gases on the 
MWCNT material properties was examined using a TIN-TEK FlexStream gas standards generator, which is capable of providing an extremely stable and accurate mix of gas at extremely low concentrations using gas permeation tubes of various gas delivery rates.

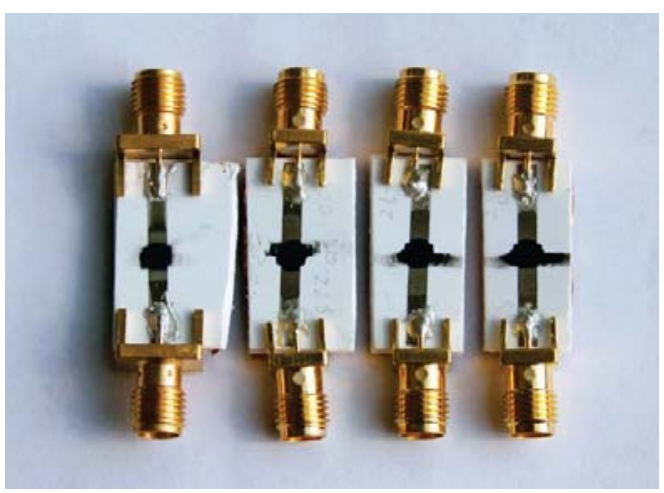

Fig. 7. CNT test samples of 25, 50, 75, and 100 layers (left to right).

Sensitivity is defined as the change of the material's impedance in presence of the test gas relative to air, over that of air. This allows the isolation of changes in material properties due to gas effects from variances in fabrication, etc, by comparing the impedance changes in a given sample solely against the inert properties of the sample. The testing procedure for both concentration and timing response at room temperature environments involved impedance measurements of 75-layered MWCNT samples taken in air and in the presence of different $\mathrm{NH}_{3}$ and $\mathrm{NO}_{2}$ concentrations, i.e. 10, 15, 20, 30, 50, 70 and $90 \mathrm{ppm}$. Especially regarding $\mathrm{NH}_{3}$, concentrations of 4 and $5 \mathrm{ppm}$ were also measured; this was not possible for $\mathrm{NO}_{2}$ due to limitations of the only available particular gas permeation tube. The samples were cleansed of impurities by placing them under pure nitrogen for 5 minutes.
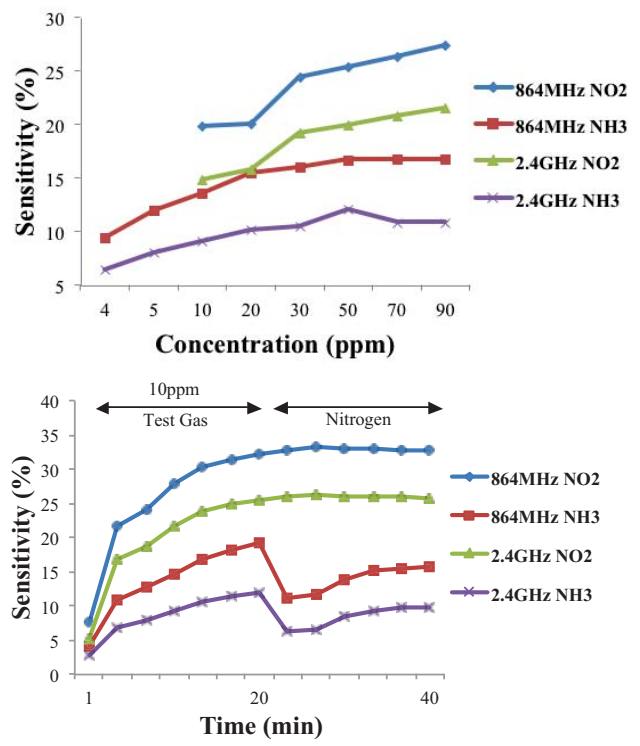

Fig. 8. (a) Response as a function of concentration taken after stabilization and (b) Timing response for $\mathrm{NH}_{3}$ and $\mathrm{NO}_{2}$ using concentration of $10 \mathrm{ppm}$ at $864 \mathrm{MHz}$ and $2.4 \mathrm{GHz}$.
Select results are provided in Fig. 8 below. First, Fig. $8 \mathrm{a}$ shows the change in sensitivity as a function of concentration for both gases at the non-licensed spectrum bands of $864 \mathrm{MHz}$ and $2.4 \mathrm{GHz}$. Sensitivity of $21.7 \%$ and $9.4 \%$ was achieved for $10 \mathrm{ppm} \mathrm{NO}_{2}$ and $4 \mathrm{ppm} \mathrm{NH}$, respectively at $864 \mathrm{MHz}$; higher than that achieved in recent work [4] for higher concentration (100 ppm) of $\mathrm{NH}_{3}$. Fig. $8 \mathrm{~b}$ provides the timing response to the same chemical gases at the chosen frequencies. For this portion of the experiment, $10 \mathrm{ppm}$ was used as the concentration for both gas tests. The measurements were taken at $1,2,5,10,15$ and 20 minutes. As shown in Fig. 8b, the MWNT-based gas sensor demonstrates fast response to both gases (few seconds); the sensitivity achieved at $864 \mathrm{MHz}$ is $24.2 \%$ for $\mathrm{NO}_{2}$ and $12.7 \%$ for $\mathrm{NH}_{3}$ in just 2 minutes' time. Note that after testing, the sensor exposed to $\mathrm{NH}_{3}$ shows more rapid recovery, possibly due to the greater electro-negativity of one pole of the $\mathrm{NH}_{3}$ molecule. Recent related work has only shown comparable time response results with significantly lower sensitivity [5].

\section{CONCLUSION}

In this paper we have presented three different types of novel smart skin structures that can accurately and reliably authenticate the identity of physical entities and provide object seal proof, detect fractures and cracks on large metallic structures and sense very low concentrations of harmful toxic gases, such as $\mathrm{NH}_{3}$ and $\mathrm{NO}_{2}$. Among their demonstrated unique characteristics are their low profile, wireless and completely passive operation, low cost of manufacturing and ultra-high sensitivity.

The aforementioned unique characteristics, coupled with their high performance in terms of accuracy and reliability, render these three types of smart skins ideal candidates and true enablers of the pervasive environmental awareness vision.

\section{ACKNOWLEDGMENT}

The authors would like to acknowledge the support of IFC-SRC. The authors would also like to thank Dr. C. P. Wong's group for providing the MWCNT material and Dr. Judy Song of GTRI for providing the gas generator.

\section{REFERENCES}

[1] V. Lakafosis, A. Traille, L. Hoseon, E. Gebara, M. M. Tentzeris, G. R. DeJean, and D. Kirovski, "RF Fingerprinting Physical Objects for Anticounterfeiting Applications," Microwave Theory and Techniques, IEEE Transactions on, vol. 59, pp. 504-514, 2011.

[2] V. Lakafosis, A. Traille, L. Hoseon, E. Gebara, M. M. Tentzeris, G. DeJean, and D. Kirovski, "RFID-CoA: The RFID tags as certificates of authenticity," RFID (RFID), 2011 IEEE International Conference on, pp.207-214, 12-14 April 2011

[3] X. Yi, T. Wu, Y. Wang, R. T. Leon, M. M. Tentzeris, and G. Lantz, "Passive wireless smart-skin sensor using RFID-based folded patch antennas," International Journal of Smart and Nano Materials, vol. 2, pp. $22-38,2011$.

[4] R. Mangu, S. Rajaputra and V. P Singh, "MWCNT-polymer composites as highly sensitive and selective room temperature gas sensors," Nanotechnology Issue 21, 2011.

[5] M. Guo, K. Wu, Y. Xu and R. Wang and M. Pan, "Multi-walled carbon nanotube-based gas sensor for NH3 Deteection at Room Temperature," ICBBE, 2010 4th International Conference on , pp.1-3, 201. 